\title{
Contrast-enhanced MRI features in the early diagnosis of Juvenile Idiopathic Arthritis
}

\author{
Robert Hemke $^{1}$ - Taco W. Kuijpers ${ }^{2}$ - Charlotte M. Nusman ${ }^{1,2}$. \\ Dieneke Schonenberg-Meinema ${ }^{2}$ - Marion A. J. van Rossum ${ }^{2,3}$ • Koert M. Dolman ${ }^{3,4}$. \\ J. Merlijn van den Berg ${ }^{2,3} \cdot$ Mario Maas ${ }^{1}$
}

Received: 26 August 2014 /Revised: 3 March 2015 /Accepted: 30 March 2015 / Published online: 23 May 2015

(C) The Author(s) 2015. This article is published with open access at Springerlink.com

\begin{abstract}
Objectives To determine whether clinical, laboratory or Magnetic Resonance Imaging (MRI) measures differentiate Juvenile Idiopathic Arthritis (JIA) from other forms of active childhood arthritis.

Materials and methods We prospectively collected data of 80 treatment-naïve patients clinically suspected of JIA with active non-infectious arthritis of (at least) one knee for $<12$ months duration. Upon presentation patients underwent clinical and laboratory assessments and contrast-enhanced MRI. MRI was not used as a diagnostic criterion.

Results Forty-four (55\%) patients were clinically diagnosed with JIA, whereas in $36(45 \%)$ patients the diagnosis of JIA was discarded on clinical or laboratory findings. MRI-based synovitis was present in $27(61.4 \%)$ JIA patients and in 7 (19.4\%) non-JIA patients $(P<0.001)$.

Five factors (male gender, physician's global assessment of overall disease activity, joints with limited range of motion, HLA-B27, MRI-based synovitis) were associated with the onset of JIA. In multivariate analysis MRI-based synovitis proved to be independently associated with JIA (OR 6.58,
\end{abstract}

Robert Hemke

r.hemke@amc.nl

1 Department of Radiology Academic Medical Center, University of Amsterdam, Meibergdreef 9, 1105AZ Amsterdam, The Netherlands

2 Department of Pediatric Hematology, Immunology, Rheumatology and Infectious Disease, Emma Children's Hospital AMC, University of Amsterdam, Meibergdreef 9, Amsterdam, The Netherlands

3 Department of Pediatric Rheumatology, Reade, Dr. Jan van Breemenstraat 2, Amsterdam, The Netherlands

4 Department of Pediatrics, St. Lucas Andreas Hospital, Jan Tooropstraat 164, Amsterdam, The Netherlands
$95 \%$ CI 2.36-18.33). In patients with MRI-based synovitis, the RR of having JIA was 3.16 (95\% CI 1.6-6.4).

Conclusions The presence of MRI-based synovitis is associated with the clinical onset of JIA. Physical examination could be supported by MRI, particularly to contribute in the early differentiation of different forms of non-infectious childhood arthritis.

Key points

- Juvenile Idiopathic Arthritis (JIA) is a diagnosis of exclusion.

- Differentiating JIA and other forms of childhood arthritis can be difficult.

- MRI-techniques have substantially improved evaluation of joint abnormalities in JIA patients.

- MRI-based synovitis is significantly associated with the clinical onset of JIA.

- MRI could support physical examination in the early differentiation of childhood arthritis.

Keywords Juvenile Idiopathic Arthritis · Childhood arthritis · Magnetic Resonance Imaging · Knee joint · Synovitis

\section{Introduction}

Juvenile idiopathic arthritis (JIA) is an umbrella term for all forms of arthritis of unknown aetiology and pathophysiology that begin before the age of 16 and persists for more than six weeks [1]. The term JIA includes seven well-defined disease categories, marked by distinct presentations, clinical features, and, in some cases, genetic backgrounds. Before JIA can be diagnosed, other known causes of arthritis in children have to be excluded. Even though JIA is a group of well-defined disease categories [1], it is sometimes hard to differentiate between JIA and other forms of childhood arthritis. Since JIA 
needs another treatment strategy than - for example - reactive arthritis or transient non-infectious arthritis [2], it is essential to discriminate the different forms of arthritis in the early phase. Moreover, early therapeutic intervention improves long-term outcome in JIA patients [3, 4]. A delay in treatment of JIA could lead to irreversible damage of the joint. Thus, a swift diagnosis and early treatment of JIA leads to a decrease in disease burden. Currently, no evidence exists for diagnostic tools that differentiate early JIA from other forms of noninfectious arthritis.

In JIA, synovial proliferation and infiltration by inflammatory cells occurs in affected joints, resulting in increased secretion of synovial fluid and synovial hypertrophy. Persistent synovitis may eventually lead to articular cartilage lesions and bone erosions that are responsible for disability and reduced quality of life in JIA [5-8]. Within the past decade, the use of magnetic resonance imaging (MRI) and advances in MRI techniques have substantially improved the evaluation of joint abnormalities in JIA patients $[9,10]$. Currently, MRI is considered to be the most suitable imaging technique in this respect [10]. Although MRI has previously been shown to be more sensitive than physical examination in the detection of active joint inflammation [11-15], its ability to distinguish different forms of childhood arthritis has been questioned $[16,17]$. Therefore, the aim of our study was to determine whether clinical, laboratory or MRI measures were able to differentiate JIA patients from other forms of childhood arthritis in a patient group clinically suspected of non-infectious early arthritis.

\section{Materials and methods}

\section{Patients}

We prospectively included 80 consecutive patients in two tertiary paediatric rheumatology centres (Academic Medical Center and Reade, both Amsterdam, the Netherlands) over a three-year period (January 2009 - December 2011). At the time of presentation, all patients had extensive clinical and laboratory assessments, followed by contrast-enhanced MRI. Inclusion criteria were clinically active arthritis suspected for JIA, involvement of at least one knee (as the most common clinically affected joint in JIA [18]), and disease duration of more than six weeks but less than 12 months. Exclusion criteria consisted of a history of intra-articular corticosteroid injection within the last six months, the need for anaesthesia during MRI, general contraindications for MRI, and arthritis based on an infection (streptococcal disease, Lyme disease or parvo infections), and $<8$ years of age (following the advice of the local medical ethical board). This study was performed in accordance with the Declaration of Helsinki and the local medical ethical regulations. Written informed consent was obtained from at least one parent of each child.

\section{Clinical and laboratory assessments}

Physical examination was performed by the same paediatric rheumatologists for each patient (JMvdB, DSM, MAJvR, TWK, and KD) during the research period. Clinical assessment included a 67-joint count defining the presence of swelling, pain on motion/tenderness, and limited range of motion. A physician's global assessment of overall disease activity, a patient's global assessment of overall well-being and an assessment of patient's pain were all measured on a $100 \mathrm{~mm}$ visual analogue scale (VAS). Functional ability was evaluated by the Dutch version of the childhood health assessment questionnaire (CHAQ) [19, 20]. Laboratory tests included the erythrocyte sedimentation rate (ESR), the C-reactive protein (CRP) level, and the presence of antinuclear antibody (ANA), human leukocyte antigen (HLA)-B27, and IgM rheumatoid factor (RF).

\section{MRI protocol and image analysis}

Contrast-enhanced MR images were obtained using 1.0-T magnet (Panorama HFO, Philips Medical Systems, Best, the Netherlands). To increase feasibility and patient comfort, we used an open-bore magnet [14]. The unsedated children were placed in the supine position with the knee joint centrally in the magnetic field using a dedicated knee coil. MRI of the clinically most affected knee (target joint) was performed. MRI sequences included sagittal T2-weighted fat saturated images (TR 2800-4500 ms; TE $50 \mathrm{~ms}$; slice thickness $4 \mathrm{~mm}$; field of view $150 \times 150 \mathrm{~mm}$; matrix $300 \times 242$ ), coronal T2weighted fat saturated images (TR 2800-4500 ms; TE $50 \mathrm{~ms}$; slice thickness $4 \mathrm{~mm}$; field of view $150 \times 150 \mathrm{~mm}$; matrix $300 \times 247$ ), axial T2-weighted fat saturated images (TR 2800-4500 ms; TE $50 \mathrm{~ms}$; slice thickness $4 \mathrm{~mm}$; field of view $150 \times 150 \mathrm{~mm}$; matrix $300 \times 270$ ), sagittal T1-weighted images obtained before and after intravenous contrast injection (TR 450-650 ms; TE $10 \mathrm{~ms}$; slice thickness $4 \mathrm{~mm}$; field of view $150 \times 150 \mathrm{~mm}$; matrix $300 \times 248,0.1 \mathrm{mmol}$ per kilogram of body weight gadobutrol (Bayer healthcare, Berlin, Germany) with an injection rate of $3 \mathrm{ml} / \mathrm{sec}$ by using an automatic injection device (Medrad, Warrendale, PA, USA)), and axial T1weighted fat saturated images obtained after intravenous contrast injection (TR 400-750 ms; TE $10 \mathrm{~ms}$; slice thickness $4 \mathrm{~mm}$; field of view $150 \times 150 \mathrm{~mm}$; matrix $272 \times 192$ ).

The image sets were scored by a radiology expert blinded to the clinical data (RH, five years of experience in musculoskeletal radiology). The MRI data sets were scored in accordance with the semi-quantitative Juvenile Arthritis MRI Scoring (JAMRIS) system. This scoring method has been validated and described before in detail [21]. Briefly, synovial 
hypertrophy was scored when thickness of the contrastenhanced synovial membrane was $\geq 2 \mathrm{~mm}$. Bone marrow changes suggestive of bone marrow oedema were defined as lesions within the trabecular bone, with ill-defined margins and high signal intensity on $\mathrm{T} 2$-weighted fat-saturated images, and low signal intensity on T1-weighted images. A cartilage lesion was defined as superficial loss and/or thinning, or deep loss to subchondral bone. Bone erosions were defined as sharply marginated bone lesions, with correct juxta-articular localization, and visible in two planes with a cortical break in at least one plane. On T1-weighted images, there is a loss of normal low signal intensity of cortical bone and loss of normal high signal intensity of trabecular bone. Synovial hypertrophy was scored at six locations: patellofemoral, suprapatellar recesses, infrapatellar fat pad, adjacent to the anterior- and posterior cruciate ligaments, medial posterior-condylar, and lateral posterior-condylar. Bone marrow changes, cartilage lesions, and bone erosions were scored at eight locations: lateral patella, medial patella, medial femur condylar, lateral femur condylar, medial weight-bearing region of the femur, lateral weight-bearing region of the femur, medial tibia plateau, and lateral tibia plateau.

\section{Study outcome}

The primary study outcome was a clinical diagnosis of JIA. According to the ILAR criteria, JIA was defined as clinical arthritis of unknown aetiology and pathophysiology that begins before the age of 16 years and persists for at least six weeks [1]. Clinical arthritis was defined as joints with swelling as well as joints with pain on motion and/or limited range of motion [1]. According to the ILAR criteria, the eventual confirmation of a definite diagnosis of JIA was made - by the same paediatric rheumatologist for each patient - after a minimal follow-up period of six months [1]. JIA is a clinical diagnosis and the diagnosis was made by the paediatric rheumatologist according to the abovementioned definitions. Solely clinical and laboratory assessments were used by the treating physician to diagnose JIA and differentiate JIA from other forms of childhood arthritis. Thus, the findings on MRI were not used in the diagnosis of JIA.

The reading radiologist was blinded for the clinical diagnosis. However, in the event that MRI findings indicated a diagnosis other than JIA (e.g., traumatic or orthopaedic pathology), the treatment strategy was adjusted by the treating physician following feedback from the radiologist.

\section{Statistics}

All data were analysed by using IBM SPSS version 19.0 (SPSS, Chicago, USA). Descriptive statistics were reported in terms of percentages, means, medians, inter-quartile ranges, and standard deviations. The Mann-Whitney $U$ test and
Fisher's exact test were used to analyse differences between groups/scores. Factors potentially associated with the study outcome in univariate analysis $(P$ value less than 0.2$)$ were included in a multivariate model. Backward stepwise selection was performed. A two-tailed $P$ value of less than 0.05 was considered statistically significant. Results were presented as odds ratios (OR) with $95 \%$ confidence intervals $(95 \% \mathrm{CI})$. Additionally, the negative predictive value (NPV), the positive predictive value (PPV), and the relative risk (RR) with $95 \%$ CI were calculated.

\section{Results}

\section{Patients}

In this prospective study, MRI datasets of 80 treatment naïve patients ( $60 \%$ female patients, mean age 12.5 years (SD 3.5)) with clinically active arthritis of the knee suspected of JIA were collected. The baseline characteristics are depicted in Table 1. A definite clinical diagnosis of JIA was made in 44 (55\%) patients according to the ILAR criteria. As a consequence, 36 (45\%), patients had no JIA. Of these non-JIA patients, $16(44.4 \%)$ were diagnosed with reactive knee

Table 1 Patient characteristics ${ }^{\mathrm{a}}$

\begin{tabular}{ll}
\hline Clinical feature & $\begin{array}{l}\text { Suspected JIA } \\
n=80\end{array}$ \\
\hline No. (\%) of female patients & $48(60.0 \%)$ \\
Age at study visit, mean years (SD) & $12.5(3.5)$ \\
Disease duration at study visit, years & $0.4(0.3-0.7)$ \\
Physician's global assessment of overall & $30(16-41)$ \\
$\quad$ disease activity & $49(20-62)$ \\
Patient's global assessment of overall & \\
$\quad$ well-being & \\
Patient's pain assessment & b \\
C-HAQ score & $59(35-74)$ \\
No. of active joints & $1.125(0.625-1.750)$ \\
No. of joints with limited range of motion & $5(1-4)$ \\
Laboratory measure & $1(0-2)$ \\
$\quad$ Erythrocyte sedimentation rate, mm/hour & $5(2-8)$ \\
C-reactive protein level, mg/l & $1(0-1)$ \\
No. (\%) of ANA-positive patients ${ }^{\mathrm{d}}$ & $25(31.3 \%)$ \\
$\quad$ No. (\%) of HLA-B27-positive patients & $18(22.5 \%)$ \\
No. (\%) of RF-positive patients &
\end{tabular}

a Except where otherwise indicated, values are median (inter-quartile range)

${ }^{\mathrm{b}}$ Measured on a $0-100 \mathrm{~mm}$ visual analogue scale $(0=$ best, $100=$ worst $)$

${ }^{c}$ Units; $0.000=$ best, $3.000=$ worst

d ANA=antinuclear antibody

${ }^{\text {e }}$ HLA-B27=human leukocyte antigen (HLA)-B27

${ }^{\mathrm{f}} \mathrm{RF}=\mathrm{IgM}$ rheumatoid factor 
symptoms (seven reactive knee symptoms based on hypermobility, four due to relative overuse, three with patello-femoral maltraction, one with large cystic lesion, and one with a retro-patellar cartilage defect); three $(8.3 \%)$ with osteochondritis dissecans, seven (19.4\%) patients with another immunologic disorder (four with systemic lupus erythematosus, one with scleroderma, one with juvenile dermatomysositis, and one with an unclassified immunologic disorder); two (5.6\%) with transient arthritis, and eight (22.2\%) patients were diagnosed with anatomical, traumatic, or other non-rheumatologic ailments (two with a meniscal tear, one with a discoid meniscus, one with a large anterior cruciate ligament ganglion, and four with a chronic pain syndrome). Concerning the JIA patients, frequencies of JIA subtypes were as follows: eight $(18.2 \%)$ persistent oligoarthritis, five $(11.4 \%)$ extended oligoarthritis, 14 (31.8\%) polyarthritis, four $(9.1 \%)$ psoriatic arthritis, ten $(22.7 \%)$ enthesitis-related arthritis, and three $(6.8 \%)$ undifferentiated JIA. Of all patients, $42(52.5 \%)$ patients received no medication, while $38(47.5 \%)$ patients used NSAIDs only.

\section{Clinical findings}

No significant differences were found between JIA and nonJIA patients regarding age (12.7 years vs. 12.3 years, $P=$ 0.961 ), physicians' global assessment of overall disease activity (34 vs. $26, P=0.053$ ), patient's pain assessment ( 62 vs. 52 , $P=0.402$ ), patient's global assessment of overall well-being (50 vs. $49, P=0.615)$, CHAQ scores ( 1.250 vs. $0.625, P=$ $0.193)$, the number of actively inflamed joints ( 2 vs. $2, P=$ 0.123 ), and the number of joints with limited range of motion ( 1 vs. $0, P=0.069$, respectively).

\section{Laboratory findings}

ESR did not differ between JIA and non-JIA patients (5 vs. 5, $P=0.157)$. There was no difference in ANA-positivity between the two groups $(29.5 \%$ vs. $33.3 \%, P=0.810)$. No
RF-positive patients were identified in this group; therefore, this parameter was omitted for further analysis.

In contrast, a significant difference between both groups was observed regarding the median CRP level (5.2 vs. 1.4, $P=0.046)$ and the number of HLA-B27-positive patients ( $31.8 \%$ vs. $11.1 \%, P=0.033$, respectively).

\section{MRI findings}

Synovial hypertrophy as a major MRI feature of synovitis was present in $27(61.4 \%)$ of the JIA patients, whereas synovial hypertrophy was present in seven $(19.4 \%)$ of the non-JIA patients $(P<0.001)$ (Figs. 1 and 2$)$. In case of non-JIA patients, their disease activity scores, joint scores, CHAQ scores, and blood parameters of inflammation (ESR and CRP) at baseline were all comparable between the non-JIA patients with or without MRI-based synovial hypertrophy. Of these seven non-JIA patients with synovial hypertrophy, three patients were diagnosed with reactive symptoms with an anatomical substrate (one based on discoid meniscus, one based on a meniscal tear, and one based on a large anterior cruciate ligament ganglion), two with osteochondritis dissecans, one patient with systemic lupus erythematosus, and one patient with transient arthritis of undetermined aetiology.

No significant differences were observed between patients with an eventual diagnosis of JIA and the non-JIA patients regarding the presence of bone marrow changes (suggestive of oedema), cartilage lesions, or bone erosions. Cartilage lesions were present in three out of 36 $(8.3 \%)$ of the non-JIA patients, including one with an abnormality interpreted as a bone erosion. Of these three patients, two were diagnosed by MRI with osteochondritis dissecans (Fig. 3), and one with reactive knee symptoms based on a meniscal tear. Two out of these three patients had accompanying MRI-based synovial hypertrophy.

Additionally, no differences were observed between the different subtypes of JIA regarding the presence of MRIbased synovial hypertrophy or bone marrow changes suggestive of bone marrow oedema.

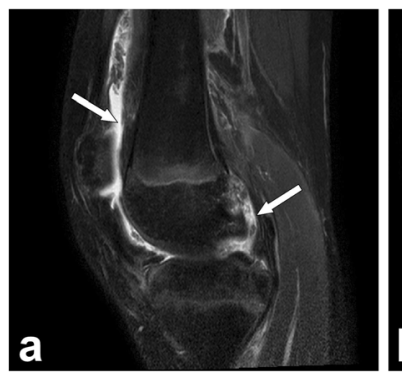

Fig. 1 Eventual JIA patient with synovitis. a. sagittal fat saturated T2weighted image, b. sagittal T1-weighted image with the injection of Gd, and an axial T1-weighted fat saturated MR image after the injection of Gd

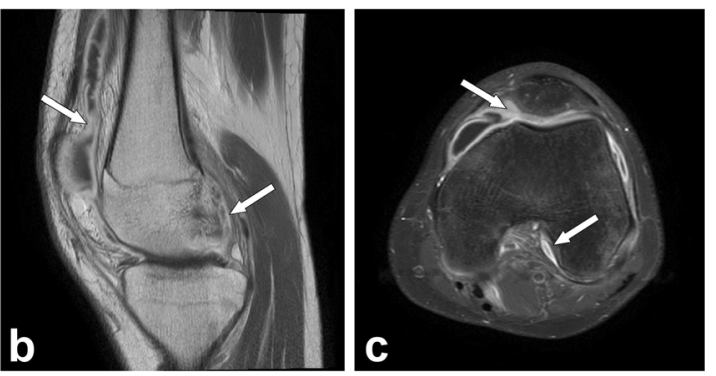

obtained in a 15-year-old boy with suspected JIA show a thickened, irregular synovial membrane (arrows) 

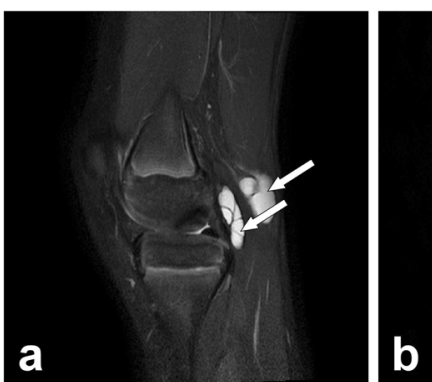

Fig. 2 Non-JIA patient with reactive knee symptoms based on a large cystic lesion. a. sagittal fat saturated T2-weighted image, b. sagittal T1weighted image with the injection of Gd, and an axial T1-weighted fat

\section{Univariate analysis}

Univariate analysis identified five factors that were potentially associated with the onset of JIA (Table 2). These were male gender (OR 3.29, $95 \%$ CI 1.26-8.57), the physician's global assessment of overall disease activity (OR 1.03, $95 \%$ CI 1.001.05), number of joints with limited range of motion (OR $1.20,95 \%$ CI 0.96-1.50), presence of HLA-B27 (OR 3.73, $95 \%$ CI 1.10-12.62), and the presence of MRI-based synovitis (OR 6.58, 95 \% CI 2.36-18.33).

\section{Multivariate analysis}

Multivariate analysis with backward selection showed that only the presence of MRI-based synovitis proved to be independently and significantly associated with the onset of JIA. In patients with the presence of MRI-based synovitis at baseline, the RR of having JIA was 3.16 (95 \% CI 1.56-6.39), the positive predictive value 0.79 , and the negative predictive value 0.63 .

\section{Discussion}

Our study evaluated whether clinical, laboratory, or MRI measures can differentiate JIA with active childhood arthritis from

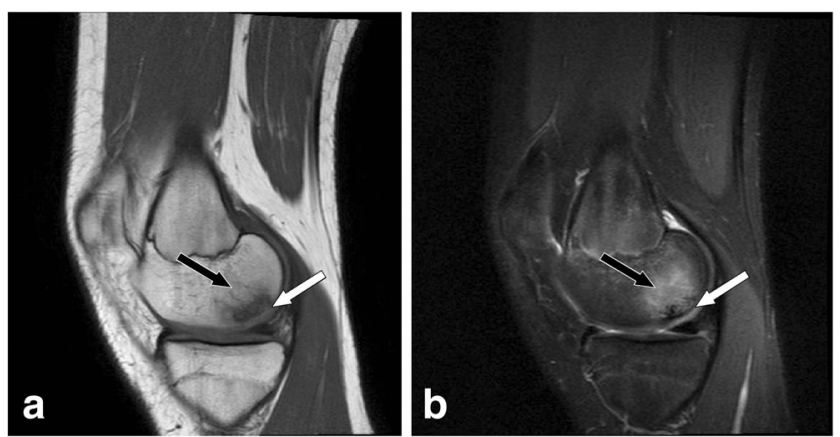

Fig. 3 Osteochondritis dissecans. a. sagittal T1-weighted post-contrast, and $\mathrm{b}$. sagittal fat saturated T2-weighted images obtained in a 15 -year-old girl show a lesion in the distal femoral condyle, with marked bone marrow oedema (black arrows) and signal intensity changes of the adjacent cartilage (white arrows) other causes of non-infectious arthritis in a group of patients with clinical signs of early arthritis. For the first time we show that of the various parameters tested, only the presence of MRI-based synovitis was found independently and significantly associated with JIA at presentation.

As early therapeutic intervention improves long-term outcome in JIA patients, objective and accurate measures in the evaluation of disease activity are essential [3, 4]. Currently, MRI is considered to be the most sensitive imaging modality for the evaluation of early inflammatory changes in JIA, including synovitis as the most critical hallmark of disease activity in JIA, as well as the late changes of cartilage and bone $[10,22,23]$. Our study implies that current measures for the categorization of different forms of childhood arthritis, which largely rely on clinical symptoms, physical joint examination, and laboratory results, are insufficient in the early discrimination of different forms of childhood arthritis. In the current study, we showed that MRI might be helpful in aiding the differentiation JIA from other causes of knee pain/symptoms in clinically suspected non-infectious arthritis. Our results are in line with another study assessing differences in MRI findings between subgroups of recent-onset childhood arthritis [24], and supports the notion that physical examination could be supported by more sensitive tools such as MRI.

Currently, MRI is the state-of-the-art imaging modality able to visualize bone marrow changes suggestive for bone marrow oedema. In rheumatoid arthritis (RA) destruction of bone is frequently preceded by bone marrow oedema. In addition, the presence of bone marrow oedema upon MRI is a key predictor of early erosive joint damage in RA [25, 26]. In paediatric JIA patients, the value of bone marrow changes is unclear. As growing joints change anatomically, it is hard to distinguish pathologic bone marrow oedema from bone marrow changes due to normal maturation. Moreover, the prevalence of signal changes suggestive of bone marrow oedema in wrists and knees of healthy children is high [27, 28]. In the current study, the presence of MRI-based bone marrow changes had no discriminating or prognostic value and was equally present in both JIA patients and non-JIA patients ( $27.3 \%$ and $33.3 \%$, respectively). The clinical relevance of the presence 
Table 2 Factors associated with the onset of JIA: univariate analysis*

Univariate analysis

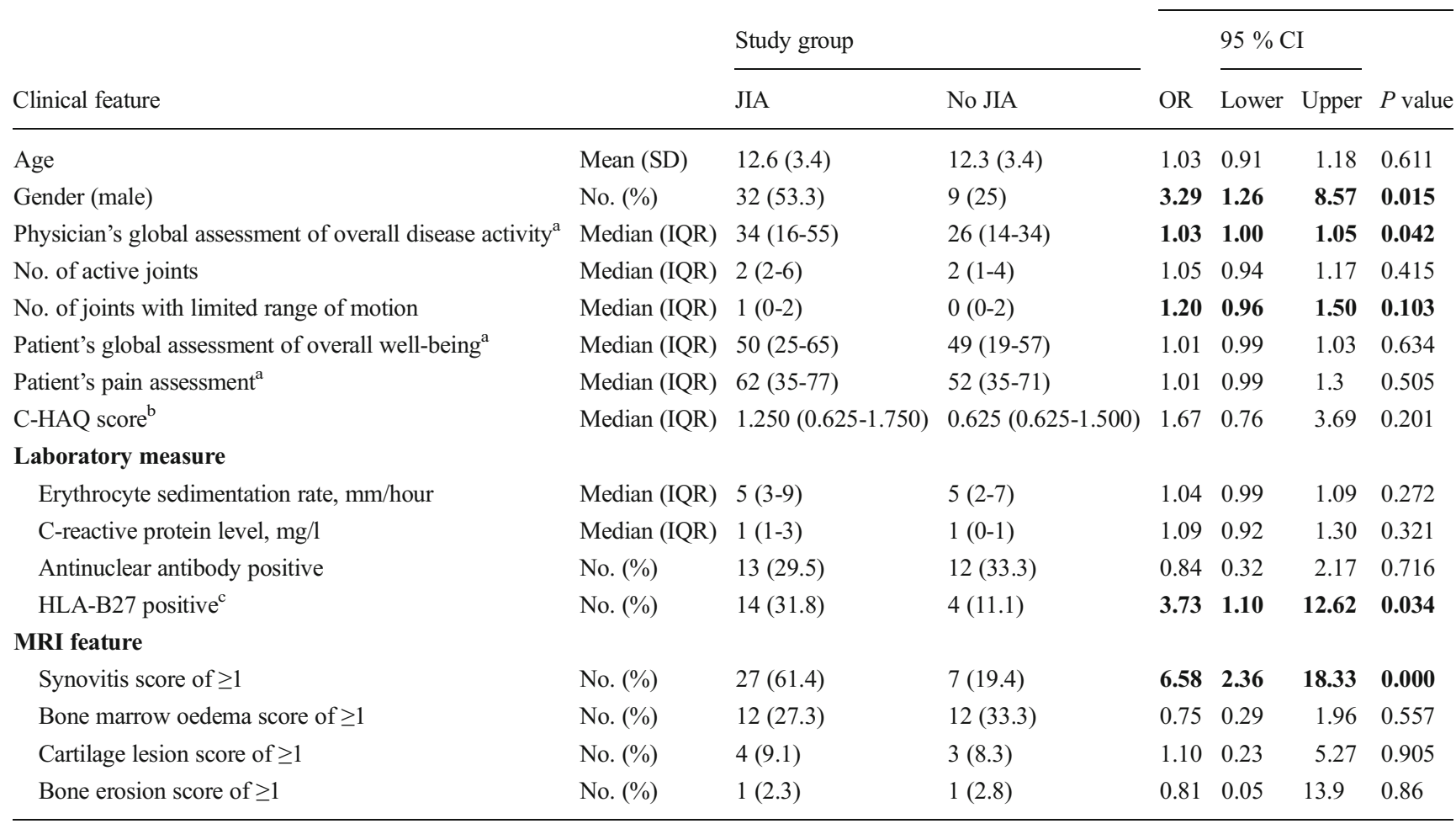

* Factors highlighted in bold are potentially associated with the onset of JIA ( $P$ value less than 0.2$)$

${ }^{a}$ Measured on a $0-100 \mathrm{~mm}$ visual analogue scale $(0=$ best, $100=$ worst $)$

${ }^{\mathrm{b}}$ Units; $0.000=$ best, $3.000=$ worst

${ }^{c}$ HLA-B27=human leukocyte antigen (HLA)-B27

of bone marrow changes in paediatric JIA patients remains, therefore, unclear. This MRI feature seems to be unrelated to JIA disease activity but is suspected to be part of the joint development or the patient's biomechanics instead.

Although MRI is the preferred imaging modality in JIA given its superior imaging quality, it has some practical limitations in daily practice. These include the necessity for sedation in very young children, the need of an intravenous contrast agent for the detection of synovial disease, and the limited number of joints that can be evaluated during one imaging session because of time constraints [10, 29]. Despite these practical limitations, the results of our current study imply that contrast-enhanced MRI is not only of value in the scope of research and clinical trials in JIA, but has important diagnostic value in daily clinical practice. Among many clinical, physical, laboratory findings taken into consideration, only the presence of MRI-based synovitis was independently and significantly associated with the onset of JIA.

Currently, we focused on MRI as an outcome measure in JIA. However, likewise the use of ultrasound (US) is being used to an increasing extent for the evaluation of disease status in JIA patients. Improvements in resolution have enhanced the performance of US. It is a safe, painless, and patient-friendly procedure without the use of ionizing radiation. Despite the advantages of US in paediatric JIA patients, it lacks standardization and information on its reliability. Moreover. the responsiveness of US to assess changes over time is unknown and US is not able to access all joints (including the knee as the most common clinically involved joint in JIA) [18, 30].

MRI is a unique method for the early distinction between JIA and other forms of childhood arthritis, and may facilitate a correct initiation and tailoring of the therapeutic strategy, given that physical examination by expert physicians seems not sufficient to guide the expert rheumatologist to an early definite diagnosis of JIA. Moreover, in the non-JIA group, signs of synovial hypertrophy upon MRI evaluation led to an alternative diagnosis that helped to avoid the improper use of antirheumatic drugs and in some cases expedited orthopaedic intervention.

The main strengths of our study consist of the prospective study design and the relatively large number of patients that were included. This is the first study assessing the predictive value of clinical, laboratory, and MRI measures to diagnose the onset of JIA in a patient group with similar clinical 
symptoms of early arthritis by clinical experts in the field. A limitation of the study is that our results may not be directly applicable to other cohorts. Moreover, the studied population may be subjected to selection bias, as the demographic and clinical findings of the enrolled patients (high mean age at disease onset, high percentage of male, and high prevalence of enthesitis related arthritis) is not a proper reflection of a real JIA population at disease onset. Besides, no differences where observed in ANA positivity between JIA and non-JIA patients, which further underpins this statement. Therefore, our results need external validation in an independent cohort to test the strength and applicability of our findings.

In summary, we conclude that the presence of MRI-based synovitis is significantly associated with the onset of JIA at the early presentation. MRI may support physical examination, particularly in the early differentiation of JIA from other forms of early non-infectious childhood arthritis and (unsuspected) orthopaedic pathology.

Acknowledgments The scientific guarantor of this publication is Prof. Dr. M. Maas, Department of Radiology, Academic Medical Center, University of Amsterdam. The authors of this manuscript declare no relationships with any companies, whose products or services may be related to the subject matter of the article. This study has received funding by a research grant that was received from the Reumafonds (NR 10-1-403); Dutch Arthritis Association (Amsterdam, the Netherlands). The Dutch Arthritis Association was not involved in designing and conducting this study, did not have access to the data, and was not involved in data analysis or preparation of this manuscript. S. Jens, MD PhD, Academic Medical Center, University of Amsterdam, kindly provided statistical advice for this manuscript. Institutional Review Board approval was obtained. Written informed consent was obtained from all subjects (patients) in this study. Methodology: prospective, diagnostic or prognostic study, multicenter study.

Funding A research grant was received from the Reumafonds (NR 101-403); Dutch Arthritis Association (Amsterdam, the Netherlands). The Dutch Arthritis Association was not involved in designing and conducting this study, did not have access to the data, and was not involved in data analysis or preparation of this manuscript.

Open Access This article is distributed under the terms of the Creative Commons Attribution-NonCommercial 4.0 International License (http:// creativecommons.org/licenses/by-nc/4.0/), which permits any noncommercial use, distribution, and reproduction in any medium, provided you give appropriate credit to the original author(s) and the source, provide a link to the Creative Commons license, and indicate if changes were made.

\section{References}

1. Petty RE, Southwood TR, Manners P et al (2004) International League of Associations for Rheumatology classification of juvenile idiopathic arthritis: second revision, Edmonton, 2001. J Rheumatol 31:390-392

2. Petty RE (1999) Prognosis in children with rheumatic diseases: justification for consideration of new therapies. Rheumatology (Oxford) 38:739-742
3. Albers HM, Wessels JA, van der Straaten RJ et al (2009) Time to treatment as an important factor for the response to methotrexate in juvenile idiopathic arthritis. Arthritis Rheum 61:46-51

4. Vilca I, Munitis PG, Pistorio A et al (2010) Predictors of poor response to methotrexate in polyarticular-course juvenile idiopathic arthritis: analysis of the PRINTO methotrexate trial. Ann Rheum Dis 69:1479-1483

5. Haverman L, Grootenhuis MA, van den Berg JM et al (2012) Predictors of health-related quality of life in children and adolescents with juvenile idiopathic arthritis: results from a web-based survey. Arthritis Care Res (Hoboken) 64:694-703

6. Magni-Manzoni S, Rossi F, Pistorio A et al (2003) Prognostic factors for radiographic progression, radiographic damage, and disability in juvenile idiopathic arthritis. Arthritis Rheum 48:3509-3517

7. Palmisani E, Solari N, Magni-Manzoni S et al (2006) Correlation between juvenile idiopathic arthritis activity and damage measures in early, advanced, and longstanding disease. Arthritis Rheum 55: 843-849

8. Oen K, Reed M, Malleson PN et al (2003) Radiologic outcome and its relationship to functional disability in juvenile rheumatoid arthritis. J Rheumatol 30:832-840

9. Johnson K (2006) Imaging of juvenile idiopathic arthritis. Pediatr Radiol 36:743-758

10. Miller E, Uleryk E, Doria AS (2009) Evidence-based outcomes of studies addressing diagnostic accuracy of MRI of juvenile idiopathic arthritis. AJR Am J Roentgenol 192:1209-1218

11. Gardner-Medwin JM, Killeen OG, Ryder CA, Bradshaw K, Johnson K (2006) Magnetic resonance imaging identifies features in clinically unaffected knees predicting extension of arthritis in children with monoarthritis. J Rheumatol 33:2337-2343

12. Nistala K, Babar J, Johnson K et al (2007) Clinical assessment and core outcome variables are poor predictors of hip arthritis diagnosed by MRI in juvenile idiopathic arthritis. Rheumatology (Oxford) 46: 699-702

13. Brown A, Hirsch R, Laor T et al (2012) Do patients with juvenile idiopathic arthritis in clinical remission have evidence of persistent inflammation on $3 \mathrm{~T}$ magnetic resonance imaging? Arthritis Care Res (Hoboken) 64:1846-1854

14. Hemke R, van Veenendaal M, Kuijpers TW, van Rossum MA, Maas M (2012) Increasing feasibility and patient comfort of MRI in children with juvenile idiopathic arthritis. PediatrRadiol 42:440-448

15. Hemke R, Maas M, van Veenendaal M et al (2014) Contrastenhanced MRI compared with the physical examination in the evaluation of disease activity in juvenile idiopathic arthritis. Eur Radiol 24:327-334

16. Buchmann RF, Jaramillo D (2004) Imaging of articular disorders in children. Radiol Clin North Am 42:151-168, vii

17. Bancroft LW (2007) MR imaging of infectious processes of the knee. Radiol Clin North Am 45:931-941, v

18. Hemke R, Nusman CM, van der Heijde DM et al (2014) Frequency of joint involvement in juvenile idiopathic arthritis during a 5-year follow-up of newly diagnosed patients: implications for MR imaging as outcome measure. Rheumatol Int. doi:10.1007/s00296-0143108-x

19. Singh G, Athreya BH, Fries JF, Goldsmith DP (1994) Measurement of health status in children with juvenile rheumatoid arthritis. Arthritis Rheum 37:1761-1769

20. Wulffraat N, van der Net JJ, Ruperto N et al (2001) The Dutch version of the Childhood Health Assessment Questionnaire (CHAQ) and the Child Health Questionnaire (CHQ). Clin Exp Rheumatol 19:S111-S115

21. Hemke R, van Rossum MA, van Veenendaal M et al (2013) Reliability and responsiveness of the Juvenile Arthritis MRI Scoring (JAMRIS) system for the knee. Eur Radiol 23:1075-1083

22. Malattia C, Damasio MB, Magnaguagno F et al (2008) Magnetic resonance imaging, ultrasonography, and conventional radiography 
in the assessment of bone erosions in juvenile idiopathic arthritis. Arthritis Rheum 59:1764-1772

23. Doria AS, Babyn PS, Feldman B (2006) A critical appraisal of radiographic scoring systems for assessment of juvenile idiopathic arthritis. Pediatr Radiol 36:759-772

24. Kirkhus E, Flatø B, Riise O, Reiseter T, Smith HJ (2011) Differences in MRI findings between subgroups of recent-onset childhood arthritis. Pediatr Radiol 41:432-440

25. Haavardsholm EA, Bøyesen P, Østergaard M, Schildvold A, Kvien TK (2008) Magnetic resonance imaging findings in 84 patients with early rheumatoid arthritis: bone marrow oedema predicts erosive progression. Ann Rheum Dis 67:794-800

26. Hetland ML, Ejbjerg B, Hørslev-Petersen K et al (2009) MRI bone oedema is the strongest predictor of subsequent radiographic progression in early rheumatoid arthritis. Results from a 2-year randomised controlled trial (CIMESTRA). Ann Rheum Dis 68: 384-390

27. Müller LS, Avenarius D, Damasio B et al (2011) The paediatric wrist revisited: redefining MR findings in healthy children. Ann Rheum Dis 70:605-610

28. Jans LB, Jaremko JL, Ditchfield M, Verstraete KL (2011) Evolution of femoral condylar ossification at MR imaging: frequency and patient age distribution. Radiology 258:880-888

29. Hemke R, Kuijpers TW, van den Berg JM et al (2013) The diagnostic accuracy of unenhanced MRI in the assessment of joint abnormalities in juvenile idiopathic arthritis. Eur Radiol 23:1998-2004

30. Collado P, Jousse-Joulin S, Alcalde M, Naredo E, D'Agostino MA (2012) Is ultrasound a validated imaging tool for the diagnosis and management of synovitis in juvenile idiopathic arthritis? A systematic literature review. Arthritis Care Res(Hoboken) 64:1011-1019 